\title{
Modeling fluid outflow from a channel consisting of three different segments
}

\author{
Kamolkhon Karimov ${ }^{1}$, Mukhiddin Khudjaev ${ }^{1, *}$, and Azamat Akhmedov ${ }^{1}$ \\ ${ }^{1}$ Tashkent State Technical University named after Islam Karimov, 2, Universitetskaya Str., 100174, \\ Tashkent, Uzbekistan
}

\begin{abstract}
The topic of the article is modeling fluid outflow from a channel consisting of three different segments. The subject of research is the outflow of fluid from a channel consisting of three different sections. The article discusses the parameters of the fluid flow in the channel segments ofthe convergingflow section, the section of a constant radius and expanding flow section. Research methods are based on Newton's rheological law; the continuity equation and the Navier-Stokes equation, which are the basic equations of fluid flow; and on the method of mathematical modeling and analytical method of solution. Expressions of hydrodynamic parameters for each segment, witha successively located converging section, a section of a constant radius, and expanding flow sections, are determined in the article by analytical method.Analytical expressions are obtained for the pressure and average flow rate of the fluid in the channel where there are converging rectilinear inlet flow area, cylindrical average area, and expanding rectilinear outlet flow area. The solutions obtained make it possible to determine the flow parameters in the zone of the vibration baffle of pipeline transport of such a geometry thatdamps vibrations caused by the flow; in the transition sections of the channels of hydraulic drives and in other channels of the fluid flow, which are set to improve the hydrodynamic parameters of the flow.
\end{abstract}

\section{Introduction}

Modeling the fluid outflow from a channel of complex geometry is one of the most important applied problems. Such areas in the pipeline transport are vibration baffles. They prevent vibrations caused by flow and are designed to lower pressure drops and to make flow patterns less separated. Such sections are set in various channels of the fluid flow to improve the hydrodynamic parameters of the flow.

A section of the channel is considered where there are converging rectilinear inlet, cylindrical middle, and expanding rectilinear outlet flow regions. In order to improve the hydrodynamic parameters of the flow, such sections are also installed in various channels for the flow of liquid media. Expressions of hydrodynamic parameters for each segment were determined by the analytical method. A quantitative analysis of the flow in these channels is conducted.

\footnotetext{
* Corresponding author: mukhiddinkhudjaev@gmail.com
} 
The outflow of fluid from successively located channels with parabolic, cylindrical and hyperbolic sections was investigated by obtaining analytical expressions for the pressure and average flow rate for each section of the channel [1].

The study of the segment in combination with elliptical tubes and spiral baffles [2] showed that geometry has a noticeable effect on the axial velocity distribution. In this case, the angle of inclination of the segment plays an important role. It turns out that the pressure drop increases at a smaller angle of inclination. Reduction in the pressure loss can be achieved by using twisted baffles instead of a segment section [3]. Comparison of this option with others showed that its overall output is higher than in the analogs with square helical tubes and cylindrical pipes.

Analyzing numerous studies aimed at improving hydraulic performance by modifying the ribs, segments with round and elliptical tubes were compared [4]. Using the methods of computer fluid dynamics to evaluate the flow patterns of six different devices, the physical reasons for the pressure drop around these devices were explained. It wasshown that pipes with elliptical cross-sections are better designed in terms of lower pressure drop and less separated flow patterns.

Modeling the flow in pipeline transport [5-8] or in segmental sections was conducted using the analytical method [8-11] and, mainly, by numerical methods with the involvement of modern information and communication systems. New methods of numerical modeling and numerical calculations are being developed. Applying these new mathematical and numerical models for a variety of problems istime-consuming work. Therefore, the problem of determining the hydrodynamic parameters in the channel segments, consisting of different rectilinear curves, is solved by the "stitching" procedure of the analytical method for solving fluid flow problems.

\section{Methods}

Research methods are based on Newton's rheological law; continuity equations and NavierStokes equations, which are the basic equations of fluid flow; on the methods of mathematical modeling and an analytical method for their solution.

\section{Materials}

Consider the fluid outflow from a segmented section, consisting of three parts:

$$
R(x)=\left\{\begin{array}{l}
R_{a} \text { at }|x| \leq x_{a}, \\
a|x|+c \text { at }|x|>x_{a} .
\end{array}\right.
$$

The center of the cylindrical part of the channel is taken as the origin of the longitudinal coordinate $\mathrm{x}$, and the direction of the channel axis from left to right is taken as a positive direction.

To obtain expressions describing changes in hydrodynamic parameters in a given channel, we rewrite the continuity equation in the following form:

$$
\frac{\partial r v}{\partial r}+\frac{\partial u r}{\partial x}=0
$$

Integrating this equation over $\mathrm{r}$ from 0 to $\mathrm{R}(\mathrm{x})$, we obtain: 


$$
\frac{\partial}{\partial x} \int_{0}^{R} u r d r=f_{1}(\mathrm{x}, \mathrm{t})
$$

From here follows the dependence for the flow rate:

$$
\frac{\partial Q}{\partial x}=f(\mathrm{x}, \mathrm{t})
$$

Where $u$ the longitudinal and radial velocity components, $P$ is the pressure, $\rho, v$-are the density and kinematic viscosity of the fluid.

Multiplying the equation of fluid flow in the divergent form [12] by $2 \pi \rho r$ and integrating over $\mathrm{r}$ from 0 to $\mathrm{R}(\mathrm{x})$, we obtain the integral equation:

$$
2 \pi \frac{\partial}{\partial x} \int_{0}^{R}\left(\rho u^{2}+P\right) r d r=\frac{\partial Q}{\partial t}+v \frac{\partial^{2} Q}{\partial x^{2}}+2 \pi R \tau_{R}
$$

Where $\tau_{R}$ is the viscous resistance force of the channel unit length on the fluid flow.

To eliminate the integral in equation (4), we replace the longitudinal component of the velocity $\mathrm{u}$ by its average flow rate $\mathrm{U}$ :

$$
\mathrm{Q}=2 \pi \rho \int_{0}^{R} u r d r=\pi R^{2} U \rho
$$

Then equation (4) takes the form:

$$
\frac{\partial}{\partial x}\left[\left(\rho u^{2}+P\right) \mathrm{R}^{2}\right]=-\rho \mathrm{R}^{2} \frac{\partial U}{\partial t}+\mu \frac{\partial^{2} R^{2} U}{\partial x^{2}}+2 R \tau_{R}
$$

Consider the stationary case. The shear stress $\tau_{\mathrm{R}}$ acting on the wall is determined approximately. Assuming that in each section the velocity profile has the form of a quadratic parabola:

$$
\mathrm{u}(\mathrm{r})=\frac{\Delta P}{4 \mu l}\left(\mathrm{R}^{2}-\mathrm{r}^{2}\right)
$$

and

$$
\mathrm{Q}=\frac{\pi \Delta P}{8 v l} R^{4}
$$

we exclude the longitudinal pressure gradient from the latter and obtain:

$$
\mathrm{u}=\frac{2 Q}{\pi \rho R^{4}}\left(\mathrm{R}^{2}-\mathrm{r}^{2}\right)
$$

This implies:

$$
\tau_{R}=-\frac{4 v Q}{\pi R^{3}}
$$


Considering that $\mathrm{Q}=\pi \rho \mathrm{R}^{2} U=\pi \rho R_{-}^{2} U_{-}$, from (5) we obtain:

$$
\frac{\partial P R^{2}}{\partial x}=-\frac{Q}{\pi} \frac{\partial U}{\partial x}-\frac{8 v Q}{\pi R^{2}}
$$

Where $\mathrm{R}_{-}, \mathrm{U}_{-}$are the input values of the channel radius and average flow rate. From (10) we determine the pressure:

$$
\mathrm{P}(\mathrm{x})=\rho \mathrm{U}\left(\mathrm{U}_{-}-\mathrm{U}+8 \mathrm{v} \int_{x_{-}}^{x} \frac{d x}{R^{2}}\right)
$$

To determine the pressure change in the inlet section of the channel, we integrate formula (11) for $R=a ! x !+c$ in this integral. The formula to determine the average flow rate follows from the equation of conservation of mass, given in integral form as $\mathrm{R}^{2} U=R_{-}^{2} U_{-}$.

$$
\mathrm{U}_{1}=\left(\frac{R-}{a x+c}\right)^{2} U_{-}
$$

For pressure:

$$
\mathrm{P}_{1}=\rho U_{1}\left[\mathrm{U}_{-}-\mathrm{U}_{1}-\frac{8 v}{a}\left(\frac{1}{a x+c}+\frac{1}{a x_{-}+c}\right)\right]
$$

For a cylindrical section of a channel of a constant radius $\mathrm{R}_{ж}$, according to the law of conservation of fluid mass, the average flow rate has the form:

$$
\mathrm{U}_{2}=\left(\frac{R_{-}}{R_{\mathscr{W}} / 2}\right)^{2} U_{-}
$$

For pressure $\mathrm{P}$ :

$$
\mathrm{P}-\mathrm{P}_{1}=-8 v \rho U_{1} \frac{x+c}{\left(R_{ж} / 2\right)^{2}}
$$

Therefore, at the end of the cylindrical channel:

$$
\begin{gathered}
\mathrm{P}_{2}-P_{1}=-16 v \rho U_{1} \frac{c}{\left(R_{ж} / 2\right)^{2}} \\
\mathrm{U}_{2}=U_{1}
\end{gathered}
$$

Integrating equation (11) for the last section, we determine:

$$
P=\rho U_{3}\left[\mathrm{U}_{-}-\mathrm{U}_{3}-\frac{8 v}{a}\left(\frac{1}{a x+c}-\frac{1}{a x_{-}+c}\right)\right]
$$

\section{Results and discussion}

Based on the study conducted, the following conclusions can be drawn: 
(1) Analytical expressions are obtained for the flow parameters in the channel, where there are a converging inlet section, a cylindrical sectionof a constant radius, and an expanding outlet section.

2. The solutions obtained make it possible to determine the flow parameters in the zone of the vibration baffle of the pipeline transport, which can be acceptedin such a geometry.

3. The obtained solutions can be used for the parameters of the working fluid in the transitional sections of the channels of hydraulic drives.

4. Analytical expressions can be used to determine the flow parameters in other channels of the fluid flow, designed to improve the hydrodynamic parametersof the flow.

\section{References}

1. K. Karimov, M.K. Khudjaev, E. Nematov, D. Khurramov, E3S Journal Conferences 224, 02003 (2020) https://doi.org/10.1051/e3sconf/202022402003

2. Wenzhu Lin, Applied Thermal Engineering 141, 164-173 (2018) https://doi.org/10.1016/j.applthermaleng.2018.05.119

3. S.M.A. Navqi Energy Procedia 158, 5770-5775 (2019) https://doi.org/10.1016/j.egupto.2019.01.533

4. Sercan Dogan, International Journal of Heat and Mass Transfer 145, 118731 (2019)

5. R.D. Enikeev, G.A. Nozdrim, A.A. Chernousov, Procedia Engineering 176, 461-470 (2017) https://doi.org/10.1016/j.proeng.2017.02.345

6. Q. Yuan, International Journal of Heat and Mass Transfer 125, 891-907 (2018) https://doi.org/10.1016/j.ijheatmasstransfer.2018.04.127

7. Jie Yang, Heng Hu, Engineering Analysis with Boundary Elements 98, 8-16 (2019) https://doi.org/10.1016/j.enganabound.2018.09.014

8. I. Khujaev, I. Arifjanov, S. Khujaeva, ISJ Theoretical \& Applied Science "Clarivate Analytics” 78(10), 25-31 (2019) https//dx.doi.org/10.15863/TAS.2019.10.78/4

9. I. Khujaev, S. Akhmadjanov, M. Khujaev, A. Ismailov, ISJ Theoretical \& Applied Science "Clarivate Analytics" $\quad \mathbf{7 8 ( 1 0 )}, \quad 32-40 \quad$ (2019) http//dx.doi.org/10.15863/TAS.2019.10.78.5

10. K.A. Karimov, M.K. Khudjaev, E.H. Nematov, T.D. Khojibekov, ISJ Theoretical \& Applied Science, "Clarivate Analytics" 82(2), 563-569 (2020) https://dx.doi.org/10.15863/TAS

11. M.K. Khudjaev, Journal of Physics: Conference Series 1614, 012093 (2020) doi:10.1088/1742-6596/1614/1/012093 